\title{
Creating trading signals using swarm technology
}

\author{
N. F. F. Ebecken \& B. S. L. P. de Lima \\ COPPE/Federal University of Rio de Janeiro, Brazil
}

\begin{abstract}
This paper focuses on the possibilities of nature inspired methods to the development of trading systems. Those systems were firstly based on neuro-genetic optimizers and indicators, and recently other alternatives have been investigated. Swarm intelligence has been shown to be attractive in different areas and motivated the development of a framework for creating trading signals. This strategy was applied in this work to a specific commodity and the results are presented.
\end{abstract}

Keywords: swarm intelligence, multi-agent systems, trading systems.

\section{Introduction}

Everybody recognizes that there is no universal trading system available, producing a perfect equity curve. Everyone has their own standards, knowledge and specific way of trading. More than that, there are infinite trading systems that do not work. Today we can consider that those trading tools are only aides.

In practice for building models (walk-forward), the well known Dr. Bandy's [1] security selection methodologies recommend one to concentrate attention on some basic points:

- Sufficient data to analyze

- The price be reasonable

- Sufficient liquidity

- Typical cycle matches the holding period and drawdown comfort level

- Enough profit potential 
Walk-forward optimization is a methodology in which the parameter values are optimized on a past segment of market data, and the system is tested forward in time on data following the optimization segment. The system is evaluated based on how well it performs on the test data, not the data it was optimized on. The process can be repeated by moving the optimization and testing segments forward in time. The premise of walk-forward optimization is that the recent past is a better foundation for selecting system parameter values than the distant past. The hope is that the parameter values chosen on the optimization segment will be well suited to the market conditions that immediately follow. The problem with this approach is that when market conditions change - say, from bull market to bear market - you may find yourself optimizing on one set of market conditions while trading a completely different set of conditions. In this case, there's no good reason to expect that the walk-forward results will be similar to the optimized results.

\section{Nature inspired methods}

In the last decade, many new stochastic search methods have been developed for the global optimization problems, such as genetic algorithms and evolutionary programming. Genetic Algorithms (GAs) are a class of stochastic search algorithms that start with a population of randomly generated candidates and 'evolve' towards better solutions by applying genetic operators (crossover, mutation, selection, etc.), modeled on the genetic processes occurring in nature. Evolutionary computation techniques have recently found many applications especially in the economic operation area.

Several neurogenetic optimizer algorithms have been proposed using numerous time series of indicators or combinations of indicators to find the most profitable models possible. During execution it is possible to see the equity curve and statistics of the models being built. The objective of the modeling can also be chosen, but generally, upward trending smooth equity curves are sought.

More recently, swarm intelligence, as demonstrated by natural biological swarms, have shown numerous powerful features that are desirable in many systems and can be applied to nonlinear and non-continuous optimization problems [3-7]. The swarm intelligence technique can generate high-quality solutions in shorter calculation time and more stable convergence characteristic than other stochastic methods.

Swarm intelligence appears in biological swarms of certain insect species and gives rise to complex and often intelligent behavior through complex interaction of thousands of autonomous swarm members.

Interaction is based on primitive instincts with no supervision. The end result is accomplishment of very complex forms of social behavior and fulfillment of a number of optimization and other tasks. The main principle behind these interactions is called stigmergy, or communication through the environment. Recently, based on the simulation of bird swarm, a particle swarm optimization (PSO) concept was developed mainly based on simulation of bird flocking in 2D space. According to the research results for a flock of birds, birds find food by 
flocking (not by each individual). The observation leads to the assumption that every information is shared inside flocking. Moreover, according to observation of human groups behavior, the behavior of each individual (agent) is also based on group decided behavior patterns such as customs and other behavior patterns based on the experiences of each individual. The position of each agent is represented by the $x y$-axis position and the velocity (displacement vector) is expressed by $v x$ (the velocity of $x$-axis) and $v y$ (the velocity of $y$-axis). Modification of the agent position is realized by using the position and the velocity information. PSO algorithm retains the conceptual simplicity of the genetic algorithm while being much easier to implement and apply to design problems with both discrete and continuous design parameters.

Particle swarm adaptation and its modifications have been shown to successfully optimize a wide range of continuous functions.

Searching procedures by PSO based on the above concept can be described as follows: a flock of agents optimizes a certain objective function. Each agent knows its best value so far and its $x y$ position. The information corresponds to the personal experiences of each agent. Moreover, each agent knows the best value so far in the group. The information corresponds to the knowledge of how the other agents around them have performed.

\section{Trading signals using swarm technology}

What are the fundamentals differences between Optimization and Adaptation?

Optimization finds the parameters that work well on history, immediately goes to "best", "best" is transient (non-stationary) and signals hop from "here" to "there" (causes discontinuities).

Adaptation adjusts parameters towards better performance, moves towards "best" tracks, "best" adjusting for transients and gracefully transitions from "here" to "there".

Based on those premises Cook [8] decided to test not a trading system, but a framework for creating trading signals using swarm technology and as such, creating a class of software referred to as "Signal Generators". Signal generators provide indications of rising and falling prices and form an important foundation for trading systems. This swarm technology provides various advantages:

- The systems and thus the signals are adaptive bar-by-bar

- Numerous alternative trading agents (bots) are used and their results combined, increasing robustness. This creates a pattern of opinions on security price movement. In more technical terms, it furnishes lateral averaging in addition to time averaging. A bot is short for "robot", an independent software entity which executes an adjustable trading system or strategy. It accepts price bar by bar and adjusts its trading system parameters and issues negative signals (sell short) and positive signals (buy) or zero valued signals (exit to cash).

- The bots, as they adapt, can communicate with each other to change their parameters to seek higher equity (referred to as "flocking"). 
- $\quad$ Bots of any type can be "added into the framework, enabling various trading styles, from Investor (long term), End of Day Trader to, eventually, Intraday Trader. A bot is a piece of software containing a trading system with changeable parameters. For example, a bot could be a moving average cross-over system with two moving averages whose periods adapt with market conditions.

This type of framework can be justified by several reasons: no trading system with a single set of parameter values is right very long averaging across parameters and signals cancels noise; increases equity and increases smoothness; "lateral" (across systems) averaging has no lag, provides an equity view across parameters and trading systems can adapt and collaborate together.

The strategy involves two types of adaptation algorithms:

- Flocking

- Bots share equity and parameter information

- Bots move towards higher performers and away from lower performers

- Bots are moving as a system

- Equity controller

- Uses industrial "PID" control algorithm

- Parameters are moved based on distance, time and rate of change of an unattainable optimum

- $\quad$ Bots are independently moving on an equity gradient

In both cases, randomness is injected to avoid "piling up" and to explore new territory.

Basically 3 concepts were considered:

- walk-forward out-of-sample represents the truth;

- $\quad$ adapt, not optimize to move and avoid brittle systems;

- committees and collections increase gains and smooth.

The development process can be summarized as follows: locate an opportunity (a ticker-bot combination), evaluate and tune the bot, build swarms, evaluate and validate the results and trade the signals and monitor performance.

The creation of trading signals using swarm technology is done in the following steps:

1. Data is extracted and bars are built (history) or bar is subscribed to

2. Data, bot, equity engine and settings are given to the swarm server

3. Swarm server clones $\mathrm{N}$ bots into a collection

4. Stops are loaded

5. Bot parameters are initialized

6. Swarm server starts stepping through the data

Each bot is provided the current bar

Each bot creates its signal

Each bot's performance is calculated and assigned back

Signal is aggregated 
Stops are evaluated and final "system" signal is created

Results are displayed

Process is repeated until stopped or data is exhausted.

The markets are always changing, thus the trading systems should too. Swarming gives parallel averaging which induces no lag. A moving average gives time-axis averaging, but there is an associated lag (e.g., 1/2 the period for simple moving averages). In a swarm, however, we also have multiple trading systems being averaged, each with slightly different parameters. This multi-bot parallelism gives a lag-less averaging effect and gives a more robust view of the operating equity surface.

\section{Case study: natural gas}

This study reports the ability to profitably trade NATURAL GAS continuous between the date 2/13/2002 and 2/6/2007 (see [2]). Twenty five bots were used and the swarm played the average signal generated from the swarm of bots employing an adaptive window slop. The adopted stop places an exit just below the rising price of long trades and just above the falling price of short trades $(3 \%$ above or below price). Figure 1 indicates the security's price, signal and equity during the period.

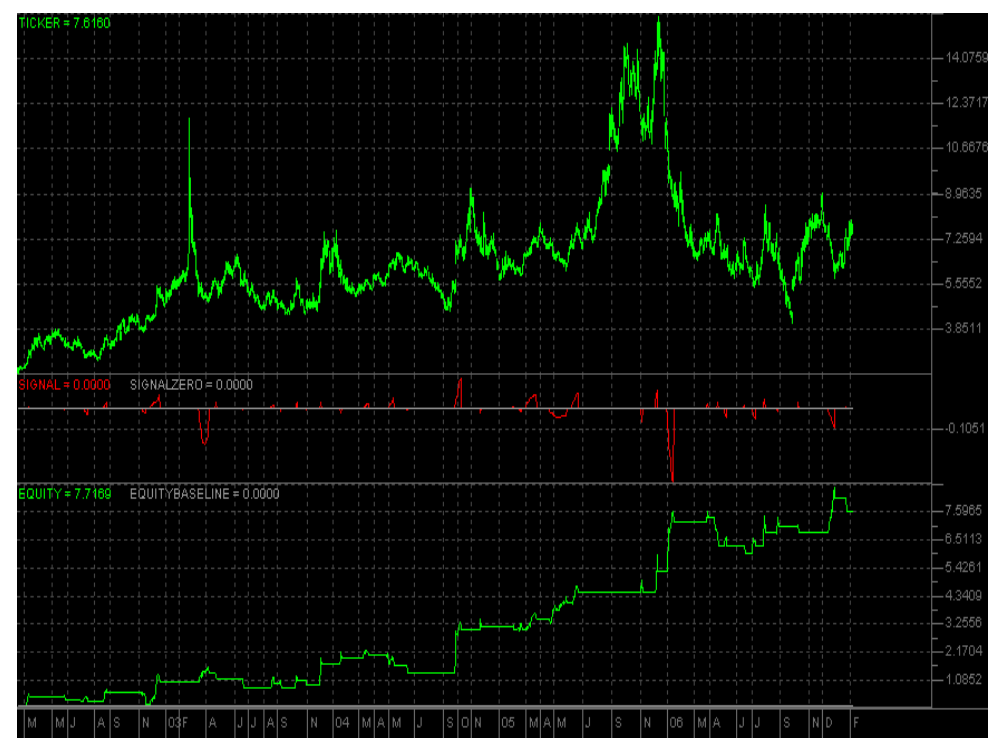

Figure 1: $\quad$ Security's price, signal and equity during the period.

The trading report for this period has indicated $58.54 \%$ winning trades, $41.46 \%$ losing trades, and 0.8947 as equity straightness. 


\section{Conclusion}

In few words, this framework presents a swarm as a collection of trading "bots" or "agents" that seek equity by varying their parameters. It takes a user-selected bot type, clones it into a swarm, tells them to initialize their trading systems' adaptable parameters, then feeds them data, one bar at a time. Next, each bot calculates its signal and the framework combines them into a "swarm-level" signal. After that, an equity performance is associated to each bot and they are able to change their trading system parameters. The bots can change their parameters by independently climbing the equity performance gradient or by cooperating with other bots (comparing parameter values vs. equity of other bots) then moving towards higher equity parameters.

Despite the fact that the calculated results can be considered with a reasonable performance, they clearly could be improved running $\mathrm{N}$ times, verifying the repeatability and observing the average performance to access the degree of "adaptation" that the solution has obtained.

The performance of the algorithm demonstrated through its evaluation that swarm adaptation on a class of software referred to as signal generators can undertake fast convergence rate and has the feature of robust computation. More than that the simulations perform very fast and it is possible to make several tries in short time.

Perhaps with the continuous use and the knowledge of the experts and analysts this technology could be incorporated as an important aide to the practice of trade activities [9].

\section{References}

[1] http://www.quantitativetradingsystems.com/issue_selection.html

[2] http://www.rydexfunds.com/

[3] Bonabeau, E., Dorigo, M., Theraulaz, G., Swarm Intelligence: from Natural to Artificial Systems. Oxford University Press, 1999.

[4] Clerc, M., Kennedy, J., The Particle Swarm-Explosion, stability, and convergence in a multidimensional complex space. IEEE Trans. on Evol. Comput., 6(1), pp.58-73, 2002.

[5] Dorigo, M., Maniezzo, V., Colorni, A., The ant system: optimization by a colony of cooperating agents. IEEE Trans. on Systems, Man, and Cybernetics, Part B, 26(1), pp 29-41, 1996.

[6] Kennedy, J., Eberhart, R.C., Particle Swarm Optimization. Proceedings of IEEE International Conference on Neural Networks, pp.1942-1948, 1995.

[7] Kennedy, J., Eberhart, R.C., Swarm Intelligence. Morgan Kaufmann Publishers, 2001.

[8] http://www.biocompsystems.com

[9] http:// www.tradestation.com 